\title{
Perioperative Patient Blood Management Programme. Multidisciplinary recommendations from the Patient Blood Management Initiative Group
}

Daniela Filipescu, Răzvan Bănățeanu, Mircea Beuran, Traean Burcoş, Dan Corneci, Dan Cristian, Mircea Diculescu, Alina Dobrotă, Gabriela Droc, Dănuț Isacoff, Doina Goşa, Ioana Grințescu, Anca Lupu, Liliana Mirea, Corina Posea, Oana Stanca, Mihai Ştefan, Dana Tomescu, Cristina Tudor, Daniela Ungureanu, Gabriel Mircescu

\section{Patient Blood Management Initiative Group}

Chairs: Professor Daniela Filipescu, MD, PhD, 'Prof. Dr. C.C. Iliescu' Emergency Institute for Cardiovascular Diseases, Bucharest; Professor Gabriel Mircescu, MD, PhD, 'Dr. Carol Davila' Clinical Nephrology Hospital, Bucharest

Secretary: Mihai Ştefan, MD, 'Prof. Dr. C.C. Iliescu' Emergency Institute for Cardiovascular Diseases, Bucharest

Members: Professor Traean Burcoş, MD, PhD, 'Colțea' Clinical Hospital, Bucharest; Associate Professor Dan Cristian, MD, PhD, 'Colțea' Clinical Hospital, Bucharest; Associate Professor Dănuț Isacoff, MD, PhD, 'Colțea' Clinical Hospital, Bucharest; Professor Anca Lupu, MD, PhD, 'Colțea' Clinical Hospital, Bucharest; Oana Stanca, MD, 'Colțea' Clinical Hospital, Bucharest; Răzvan Bănățeanu, MD, 'Floreasca' Clinical Emergency Hospital, Bucharest; Professor Mircea Beuran, MD, PhD, 'Floreasca' Clinical Emergency Hospital, Bucharest; Professor Ioana Grințescu, MD, PhD, 'Floreasca' Clinical Emergency Hospital, Bucharest; Associate Professor Liliana Mirea, MD, PhD, 'Floreasca' Clinical Emergency Hospital, Bucharest; Cristina Tudor, MD, 'Floreasca' Clinical Emergency Hospital, Bucharest; Professor Mircea Diculescu, MD, PhD, 'Fundeni' Clinical Institute, Bucharest; Associate Professor Gabriela Droc,MD, PhD,'Fundeni' Clinical Institute, Bucharest; Associate Professor Dana Tomescu, MD, PhD, 'Fundeni' Clinical Institute, Bucharest; Daniela Ungureanu, MD, 'Fundeni' Clinical Institute, Bucharest; Associate Professor Dan Corneci, MD, PhD, 'Elias' Emergency University Hospital, Bucharest; Doina Goşa, MD, Blood Transfusion Centre, Bucharest; Alina Dobrotă, MD, 'Prof. Dr. C. T. Nicolau' National Institute of Transfusion Haematology; Corina Posea, MD, 'Prof. Dr. C. T. Nicolau' National Institute of Transfusion Haematology

\begin{abstract}
Patients with untreated anaemia or iron deficiency who undergo surgical procedures have an increased risk for mortality and morbidity. Patient Blood Management programmes address this issue worldwide and try to improve patient outcomes through a complex set of measures targeting anaemia correction, minimisation of bleeding and improvement of anaemia tolerance, in all phases of perioperative care.

The Patient Blood Management Initiative Group is a multidisciplinary team of physicians from specialties including anaesthesiology, nephrology, surgery, orthopaedics, haematology, gastroenterology and transfusion medicine. The team has elaborated ten recommendations, divided into five categories, in order to implement a Patient Blood Management programme in Romania, using the most recent and relevant evidence. The document was discussed during three meetings which took place during October 2016 and May 2017 and the result was modified and updated via e-mail.
\end{abstract}

Keywords: anaemia, iron, transfusion

Received: 7 July 2017 / Accepted: 30 August 2017

Rom J Anaesth Intensive Care 2017; 24: 139-157

\section{Premises}

Patients with untreated anaemia or iron deficiency who are referred for a surgical procedure with a pre-

Address for correspondence: Dr. Mihai Ştefan Institutul de Urgență pentru Boli Cardiovasculare "Prof Dr C C Iliescu" Institutul Clinic Fundeni, Clădirea B Şoseaua Fundeni, nr 258

Sector 2, Bucureşti, Romania E-mail:mihai.steph@gmail.com sumed high bleeding risk have an increased postoperative morbidity and mortality risk $[1,2]$. Blood administration (transfusion) is not an aetiological treatment in such cases and may be associated with high morbidity [3] while unjustifiably reducing human blood components available for therapeutic use.

Back in 2010, the World Health Organisation (WHO) already had recommended the rational use of blood products by using alternative solutions to transfusion and implementation of Patient Blood Management (PBM) programmes [4]. 
PBM is a proactive, multidisciplinary, multimodal, patient-centred concept, which includes anaemia detection and treatment, haemostasis optimisation, blood loss minimisation, rational use of blood products and optimisation of anaemia tolerance, to improve the patient's outcome [5-7]. PBM is currently considered a new safety and quality standard for patient care [8].

In time, PBM has become a new standard of care, with proven evidence in multiple areas of medicine. Professionals within medicine must use PBM to improve patient outcome. Not doing so represents suboptimal patient care and may harm individuals [9].

PBM programmes are implemented across healthcare systems around the world, at hospital/institutional or national level. They seek to coordinate the hospital care that patients receive from physicians of different specialities aiming to reduce the blood products use, especially during - but also beyond - the perioperative period. They are based on four principles [5]:

1. Anaemia management

2. Coagulation optimisation

3. Use of interdisciplinary blood conservation strategies

4. Patient involvement in medical decisions related to blood transfusion

The PBM concept comprises three types of measures:

1. Patient's red cell mass optimisation

\section{Blood loss minimisation}

3. Patient's tolerance of anaemia optimisation and rational usage of therapeutic blood components [4].

These measures must be applied throughout the entire period of care of the surgical patient, being initiated preoperatively and continued intraoperatively and postoperatively (Table 1). For an easier implementation, they can be organised into "multimodal packages" for patients' blood conservation [5].

The experience of other hospitals/healthcare institutions shows that applying PBM principles throughout the entire perioperative period may decrease mortality $[12,13]$, morbidity $[12,14]$, in-hospital length of stay $[12,13]$ and costs [15], allowing for a better use of medical resources [16], while maintaining patient safety [17].

Based on this medical evidence, physicians of different specialties set up an Initiative Group to introduce the PBM concept in Romania. During three joint meetings, group members examined relevant literature and available best practice guidelines, based on their personal experience and Romanian standards of practice, and developed a set of recommendations for introducing the PBM Programme into several hospitals in Bucharest ('Prof. Dr. C.C. Iliescu' Emergency Institute for Cardiovascular Diseases, 'Fundeni' Clinical Institute, 'Floreasca' Clinical Emergency Hospital,

Table 1. Patient Blood Management Programme Components (amended based on references [6, 10, 11])

\begin{tabular}{|c|c|c|c|}
\hline & $\begin{array}{l}\text { Optimise the patient's } \\
\text { red cell mass }\end{array}$ & Minimise blood loss & $\begin{array}{l}\text { Improve patient tolerance of anaemia } \\
\text { and rationalise blood product usage }\end{array}$ \\
\hline 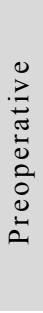 & $\begin{array}{l}\text { - } \text { Detect anaemia } \\
\text { - Identify the cause } \\
\text { - Treat the cause } \\
\text { - Manage iron deficiency } \\
\text { - Treat other haematinic } \\
\text { deficiencies } \\
\text { - Stimulate erythropoiesis } \\
\text { - Recommend specialist } \\
\text { consultation, if necessary }\end{array}$ & $\begin{array}{l}\text { - Identify and treat haemostatic abnormalities } \\
\text { - Minimise iatrogenic blood loss } \\
\text { - Plan and re-assess procedures } \\
\text { - Use autologous blood donation for selected } \\
\text { cases }\end{array}$ & $\begin{array}{l}\text { - Assess the patient's physiological reserve } \\
\text { - Optimise cardiac output through maintenance of } \\
\text { normovolemia and rational use of vasoactive } \\
\text { substances } \\
\text { - Compare estimated blood loss with the patient's } \\
\text { tolerable blood loss } \\
\text { - Plan blood conservation modalities } \\
\text { - Use restrictive transfusion protocols }\end{array}$ \\
\hline 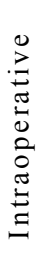 & $\begin{array}{l}\text { - Timed surgery with } \\
\text { haematological optimisation }\end{array}$ & $\begin{array}{l}\text { - Use meticulous haemostasis } \\
\text { - Use blood-sparing surgical techniques } \\
\text { - Use induced hypotension in selected cases } \\
\text { - Keep low venous pressure } \\
\text { - Use intraoperative blood salvage } \\
\text { - Use haemostatic drugs (tranexamic acid, } \\
\text { - Usmopressin) }\end{array}$ & $\begin{array}{l}\text { - Optimise cardiac output } \\
\text { - Optimise ventilation and oxygenation } \\
\text { - Use restrictive transfusion protocols } \\
\text { - Use acute normovolemic haemodilution in } \\
\text { selected cases }\end{array}$ \\
\hline 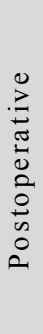 & $\begin{array}{l}\text { - Assess anaemia } \\
\text { - Manage iron deficiency } \\
\text { - Stimulate erythropoiesis } \\
\text { - Awareness of drug interactions } \\
\text { which can aggravate anaemia }\end{array}$ & $\begin{array}{l}\text { - Monitor closely haemostasis and bleeding } \\
\text { - Avoid secondary haemorrhage } \\
\text { - Assess antithrombotic prophylaxis and } \\
\text { treatment management } \\
\text { - Reduce iatrogenic blood loss } \\
\text { - Maintain normothermia } \\
\text { - Plan autologous blood conservation } \\
\text { modalities in selected cases } \\
\text { - Use prophylaxis of upper gastrointestinal } \\
\text { bleeding }\end{array}$ & $\begin{array}{l}\text { - Optimise the patient's physiological reserve } \\
\text { - Maximise oxygen delivery } \\
\text { - Minimise oxygen consumption } \\
\text { - Use restrictive transfusion protocols } \\
\text { - Avoid/Treat infections promptly }\end{array}$ \\
\hline
\end{tabular}


'Elias' Emergency University Hospital, and 'Colțea' Clinical Hospital).

The PBM Programme has the following objectives:

1. To improve the patient's outcome by:

1.1. Assessing and treating perioperative anaemia

1.2. Assessing and treating coagulation disorders

1.3. Avoiding unnecessary blood transfusion and, thus,

1.4. Reducing transfusion-associated infectious and non-infectious complications

2. To deliver blood products to patients who actually need a transfusion

3. To decrease the costs of hospital stay.

The Initiative Group's long-term objective is to implement a national PBM Programme that can improve the safety and quality of healthcare in Romania.

Firstly, the general perioperative recommendations were discussed and developed, to be followed by further recommendations concerning geriatric patients and patients with chronic kidney disease, heart failure, or haematological conditions.

The Initiative Group formulated 10 recommendations together with relevant implementation assessment indicators which are described in five chapters: I. Organisation of the PBM Programme; II. Patient red cell mass optimisation; III. Blood loss minimisation; IV. Optimisation of anaemia tolerance and rational use of blood products; V. PBM Programme monitoring and evaluation benchmarks.

\section{Organisation of the PBM Programme}

Recommendation 1. Setting up a hospital PBM Implementation Group

- In each hospital where the PBM Programme will be introduced, a PBM Implementation Group (IG) should be set up, initially on a voluntary basis, comprising anaesthesiologists, representatives of blood transfusion departments and haemovigilance committees, surgeons (with various specialties), internists, haematologists, nephrologists, physicians with other specialities, pharmacists and nurses involved in perioperative care; an IG Coordinator should be appointed.

- For PBM Programme implementation, the involved healthcare institutions should provide relevant continuous medical education.

Implementation Assessment

- Biannual reports from the hospital PBM IG

- Number of PBM related education events organised by each healthcare institution participating in the Programme.

The hospital PBM IG will:
- Develop standard operating procedures (SOPs) based on the PBM Programme recommendations

- Effectively implement the PBM Programme

- Help to define the benchmarks for evaluation of the performance

- Monitor the completeness and the accuracy of recorded data

- Report to the IG Coordinator any relevant data concerning the patients included in the PBM Programme

- Organise continuous medical education events in order to disseminate information on PBM Programme and benefits to other healthcare specialists and patients.

The IG Coordinator shall:

- Organise and chair IG meetings

- Organise the monitoring of PBM Programme implementation

- Represent the IG in relations with the hospital manager, heads of departments, and IG from other hospitals

- Present performance indicator reports to heads of department and to hospital management, on a quarterly basis

- Communicate the results of PBM Programme implementation to other IG groups and to the Initiative Group

- Assess and communicate the PBM Programme benefits to patients.

It is preferable that the IG members appoint an anaesthesiology specialist as the Coordinator. According to the definition of this specialty, which includes perioperative medicine [18], the anaesthesiologist is in the ideal position to continuously monitor the surgical patients during their hospital stay.

To ensure information sharing and increased medical staff involvement in the PBM Programme, it is important to provide educational support [5]. To this aim, regular meetings should be organised, such as conferences, symposia and workshops, to explain the PBM Programme to physicians, clinical pharmacists, trainees and nurses.

\section{Patient Red Cell Mass Optimisation}

Recommendation 2. Planning of the surgical intervention

- Anaemia and iron deficiency must be diagnosed sufficiently in advance of the elective procedures associated with a high bleeding risk (see APPENDIX 1), to allow red cell mass optimisation and reduce the need of a blood transfusion. It is recommended that surgical patients are evaluated by the anaesthe- 
siologist more than one week before the planned procedure.

- In non-elective procedures, blood products should be used rationally (see Recommendations 7-9).

Implementation Assessment

The percentage of patients evaluated by the anaesthesiologists more than one week before surgery, from the total number of patients who underwent surgical procedures with a high bleeding risk.

The risk of surgical haemorrhage must be estimated in every hospital, based on specific pathology and recent years' results. The PBM Programme will be applied to patients whose estimated perioperative blood loss exceeds $500 \mathrm{ml} \mathrm{[19].}$

Recommendation 2 can be implemented by using different pathways agreed by the IG. Generally, the physicians who indicate and/or schedule patients for surgery should refer the patients for a preoperative anaesthesiology evaluation. Ideally, preoperative evaluation should take place four weeks before surgery [20].

Recommendation 3. Assessing anaemia and iron deficiency

- The minimum tests recommended for anaemia andiron deficiency assessment are: complete blood count, serum ferritin, transferrin saturation index (TSAT - measured based on serum iron and serum transferrin levels), and C-reactive protein (CRP).

- The aim of the investigations is to identify irondeficient patients, whose red cell mass and/or iron status may be optimised through iron administration.

- If the cause of iron deficiency is unclear, additional exams/investigations should be ordered for diagnostic purposes.

- In some cases - chronic kidney disease with eGFR below $30 \mathrm{~mL} / \mathrm{min}$, rheumatic diseases, inflammatory bowel disease, blood disorders, or cancer - the recommendation for iron administration and treatment with erythropoiesis-stimulating agents requires a specialist consultation.

\section{Implementation Assessment}

The percentage of patients with anaemia and iron deficiency (absolute or functional) identified at least one week before surgery from the total number of patients who have undergone surgical procedures with a high bleeding risk.

Anaemia is a global health problem, affecting approximately $30 \%$ of the population [8]. While it can be associated with chronic diseases, $30-50 \%$ of the cases are caused by iron deficiency. Perioperatively, patients with anaemia need more blood transfusion and their outcome is less favourable $[1,2]$. Therefore, anaemic patients must be identified and treated before surgery. Not treating anaemia preoperatively is considered a substandard medical practice [9]. Iron deficiency should also be detected as it is a determining/ aggravating factor for anaemia, especially in procedures with a high bleeding risk.

The WHO defines anaemia as a haemoglobin $(\mathrm{Hb})$ level below $13 \mathrm{~g} / \mathrm{dl}$ in men and $12 \mathrm{~g} / \mathrm{dl}$ in women [21]. To diagnose anaemia, a minimum assessment should include a complete blood count (required for diagnosing anaemia), determining the levels of serum ferritin (indicator of iron stores), serum iron and serum transferrin needed to measure TSAT (an indicator of iron available for erythropoiesis and required for iron deficiency assessment) and testing the level of CRP (an indicator of inflammation, required for assessing anaemia of chronic disease) (Figure 1).

Iron deficiency should be detected and corrected by iron administration as follows:

- TSAT over $25 \%$ indicates anaemia of chronic disease and excludes the need of iron administration;

- TSAT below 25\% indicates reduction of iron available for erythropoiesis; to establish the need for iron administration, serum ferritin levels should be determined;

- Ferritin level below $100 \mathrm{ng} / \mathrm{mL}$ indicates the depletion of iron stores and, in association with a TSAT below $25 \%$, points to a diagnosis of absolute iron deficiency, in which case iron administration is recommended;

- Ferritin level over $100 \mathrm{ng} / \mathrm{mL}$ points to functional iron deficiency or iron tied up in body stores because of inflammation (anaemia of chronic disease); as ferritin is an acute phase reactant, CRP can help identify cases in which anaemia may respond to iron supplementation;

- CRP level below $10 \mathrm{mg} / \mathrm{L}$ suggests functional iron deficiency even if serum ferritin is over 100 $\mathrm{ng} / \mathrm{mL}$, in which case iron should be administered, as directed by specialist physicians.

- CRP level over $10 \mathrm{mg} / \mathrm{L}$ and ferritin level over $100 \mathrm{ng} / \mathrm{mL}$ indicate anaemia of chronic disease, in which case a specialist consultation should be conducted to establish whether iron therapy is opportune.

Note: Some patients could be iron deficient even in the absence of anaemia. Iron deficiency should be treated in these cases too, especially if the risk of perioperative bleeding is high, as bleeding aggravates iron deficiency and leads to anaemia [6].

In most cases, iron deficiency is caused by bleeding. If the source of bleeding is unknown, it should be identified through specialist consultations.

Ferritin thresholds for diagnosing functional iron deficiency may vary, depending on co-morbidities 


\section{Patients with indications for procedures associated with high risk of bleeding}

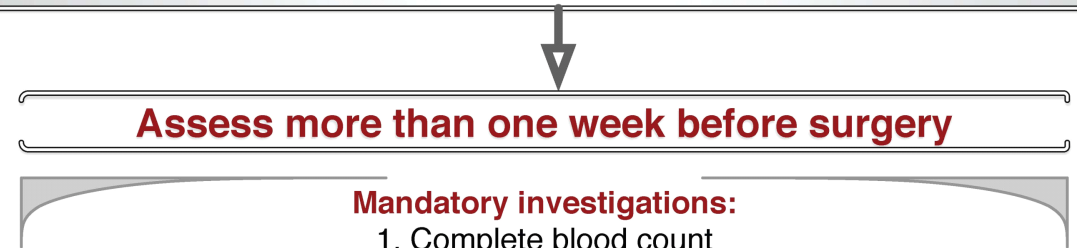

2. Serum Iron, serum transferrin $\rightarrow$ Transferrin saturation (TSAT)

3. Serum ferritin

4. C-reactive protein (CRP)
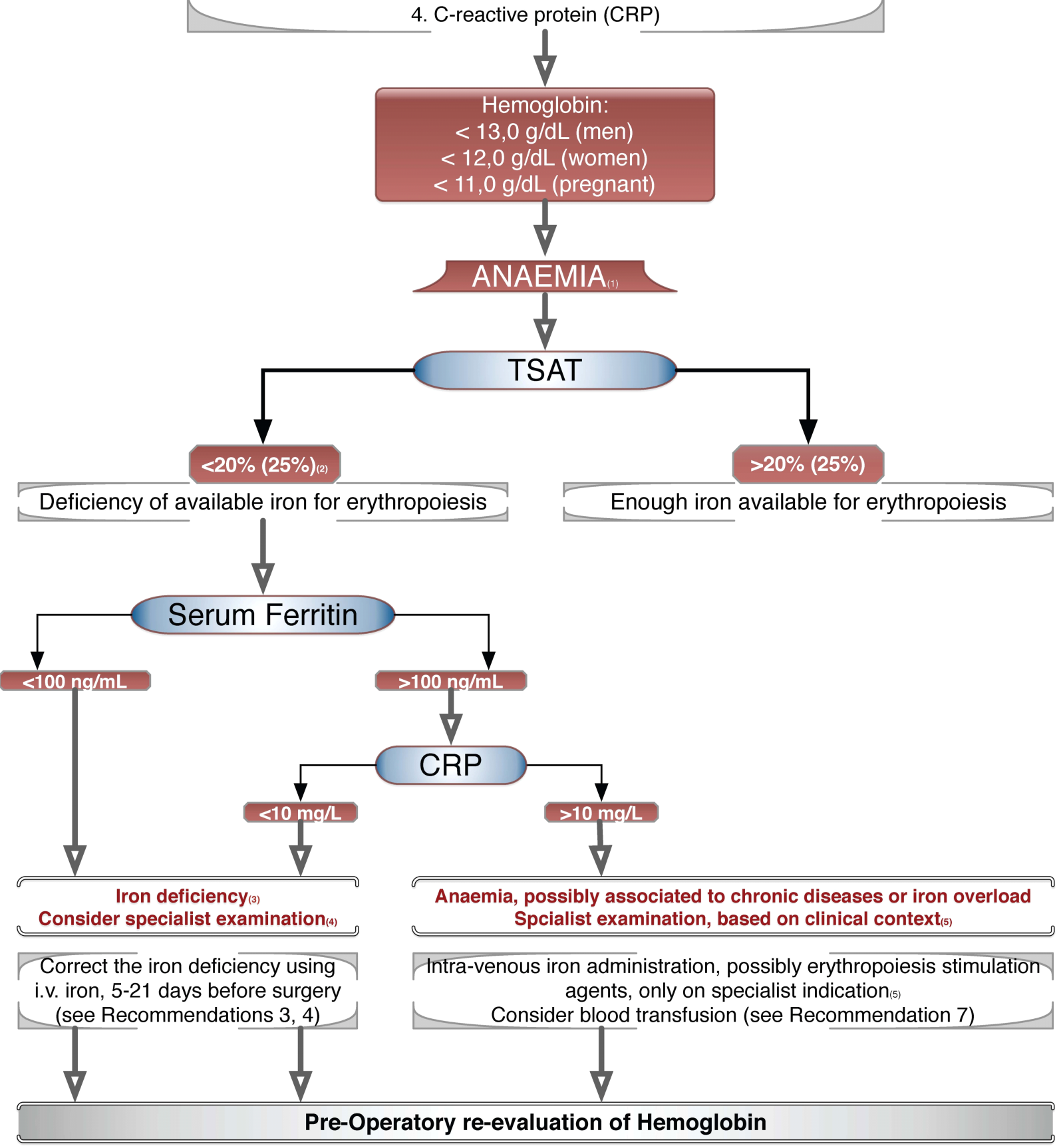

Fig. 1. Algorithm for Patient Red Cell Mass Optimisation. (1) Sometimes, specific (megaloblastic, haemolytic) anaemia may need to be addressed; (2) TSAT $<20 \%$ indicates absolute iron deficiency, while levels below $25 \%$ recommend iron therapy in cases of anaemia associated with iron deficiency and inflammation; (3) Iron deficiency may be present in the absence of anaemia and requires preoperative correction; (4) If iron deficiency has no apparent cause, it should be identified by specialist examination; (5) The need and dosage for iron supplementation and erythropoiesis-stimulating agents (ESA) are to be exclusively determined by a specialist 
(chronic kidney disease with eGFR below $30 \mathrm{~mL} / \mathrm{min}$, rheumatic diseases, inflammatory bowel disease, blood disorders, or cancer). Thus, before recommending iron administration, specialist consultations may be required based on the clinical context.

Macro-megaloblastic and haemolytic anaemias are rare and usually diagnosed before the procedure. Therefore, relevant screening tests - peripheral blood smear, reticulocyte count, lactate dehydrogenase (LDH), serum haptoglobin - are not routinely recommended. However, if these anaemias are clinically suspected, a haematology consultation should be conducted.

Recommendation 4. Optimising patient red cell mass

- In patients with absolute or functional iron deficiency, whether anaemic or not, iron should be administered intravenously (i.v.) before surgery.

- I.v. iron should be administered ideally 5-21 days before surgery; in cases where this is not possible, rescue erythropoiesis can be undertaken, and i.v. iron can be administered up until (and including) the day before surgery.

- Erythropoiesis-stimulating agents (ESAs) may help in certain clinical conditions - chronic kidney disease with eGFR below $30 \mathrm{~mL} / \mathrm{min}$, cancer, or anaemia of chronic disease - as directed by specialist physicians.

\section{Implementation Assessment}

- The percentage of patients with iron deficiency (absolute and functional) in whom iron was administered before surgery from the total number of iron deficient patients undergoing surgery with a high bleeding risk.

- The percentage of patients in whom rescue erythropoiesis was undertaken from the total number of iron deficient patients undergoing surgery with a high bleeding risk.

\section{Iron Therapy}

The aim of iron administration is to correct iron deficiency and reduce the need for blood transfusion, through haemoglobin optimisation.

Orally administered iron, in patients without contraindications, can effectively treat iron deficiency and anaemia, but only after several months, which is too slow for most surgical cases. Given the adverse effects and poor patient adherence to oral iron therapy, intravenous administration is preferable in the preoperative phase $[6,8,22]$.

Older i.v. iron preparations only allow for small quantities of iron to be administered in a single dose (200 mg of elemental iron), whereas newer preparations can be administered in higher single doses (500-
$1000 \mathrm{mg}$ ), allowing the correction of iron deficiency with one or two doses. Considering the faster effect and the safety profile, the newer iron preparations are preferable for surgical patients [19].

Practical indications for iron administration are detailed in APPENDIX 2.

Iron administration is contraindicated in patients with active infections [24].

In patients where clinical judgment deems it appropriate, supplemental folic acid and vitamin B12 should be administered, with or without concomitant iron therapy.

\section{Erythropoiesis-Stimulating Agent Therapy}

ESAs can be used in certain cases of anaemia renal anaemia, cancer, anaemia of chronic disease as recommended by specialist physicians [20,25].

Anaemia treatment principles must also be followed postoperatively.

\section{Blood Loss Minimisation}

Recommendation 5. Assessing haemostasis abnormalities

- A standardised haemostasis abnormality assessment questionnaire is recommended to be applied preoperatively for screening high bleeding risk patients (see APPENDIX 3). A positive answer to any of the questions indicates that haemostasis abnormalities need to be further evaluated by lab tests and specialist consultations (cardiology, haematology, etc.).

- Anticoagulant and antiplatelet agent therapy should be adjusted perioperatively according to the institutional protocols.

- Perioperative haemostasis management algorithms using point-of-care tests may be beneficial in procedures with a high bleeding risk.

- It is recommended to maintain optimal haemostasis conditions $(\mathrm{pH}>7.2$, normothermia, ionized serum calcium of 1.1-1.3 $\mathrm{mmol} / \mathrm{L}$ ) during the entire perioperative period.

- Tranexamic acid should be administered prophylactically in surgeries with a high bleeding risk and in cases with suspected hyperfibrinolysis and bleeding.

- The use of desmopressin is suggested in selected cases (suspicion of congenital or acquired platelet dysfunction).

\section{Implementation Assessment}

- The percentage of patients with a haemostasis abnormality risk assessment questionnaire completed, from the total number of patients with high bleeding risk surgeries.

- The percentage of patients with positive answers to the questionnaire, in whom haemostasis abnor- 
malities were detected, from the total number of patients with a positive answer.

- The percentage of patients with anticoagulant and/ or antiplatelet agents, adjusted perioperatively, from the total number of patients treated with anticoagulants and/or antiplatelet drugs who underwent procedures with a high bleeding risk.

- The percentage of patients in whom point-of-care tests were used to detect perioperative haemostasis abnormalities, from the total number of patients who underwent procedures with a high bleeding risk.

- The percentage of patients who received tranexamic acid prophylactically or as a treatment, from the total number of patients who underwent procedures with a high bleeding risk.

Routine haemostasis tests - activated partial thromboplastin time, prothrombin time, platelet count - have a poor predictive value for intra/postoperative bleeding [26]. Therefore, these tests are only recommended for patients with at least one positive answer to the standard haemostasis assessment questionnaire (APPENDIX 3). If haemostasis abnormalities are suspected, a specialist consultation should be set up (haematology, gastroenterology) $[6,27,28]$.

It is recommended that any therapy with haemostasis-interfering medication - anticoagulants, antiplatelet drugs, nonsteroidal anti-inflammatory drugs - should be adjusted perioperatively according to guidelines, following interdisciplinary evaluations [20].

Viscoelastic tests for haemostasis assessment (thromboelastography and thromboelastometry) use whole blood and may be conducted at the point of care (in the operating theatre or intensive care unit) or in the central laboratory. Algorithms based on these tests have proved helpful for intraoperative coagulopathy diagnosis and management in cardiac surgery [29]. Their use for non-cardiac surgery with a high bleeding risk can reduce the need for blood transfusion more than standard haemostasis monitoring tests [20].

Maintaining the homeostasis of the internal environment (normal $\mathrm{pH}$, temperature and serum calcium level) is essential for normal haemostasis. Any change in one or all of these parameters can lead to severe haemostasis abnormalities, causing increased perioperative blood loss $[20,30]$.

Prophylactic administration of tranexamic acid was proven to reduce blood loss in major surgery, at doses of 20 to $25 \mathrm{mg}$ per $\mathrm{kg}$ of body weight. Ongoing bleeding should be treated similarly when hyperfibrinolysis is suspected $[20,30]$.

Recommendation 6. Minimising iatrogenic blood loss
- A minimal amount of blood should be collected for lab tests and micro tubes should be used when repeated tests are needed.

- It is recommended to use closed flush systems for arterial lines and central venous catheters in patients with anaemia and/or with high bleeding risk.

- Blood salvage (cell-saver) systems should be routinely used during procedures with a high bleeding risk, in the absence of contraindications, like surgical site infection or oncologic surgery.

Implementation Assessment

- The percentage of patients for whom micro tubes were used, from all investigated patients who underwent surgical procedures with a high bleeding risk.

- The percentage of patients for whom closed flush systems were used for arterial lines and central venous catheters, from all patients with arterial lines or central venous catheters who underwent surgical procedures with a high bleeding risk.

- The percentage of patients for whom blood salvage (cell-saver) systems were used from patients who underwent surgical procedures with a high bleeding risk.

The amount of blood collected for lab tests can be significant, especially when repeated testing is prescribed. This blood loss, estimated at nearly $454 \mathrm{ml}$ per case in cardiac surgery [31], is usually neglected and may aggravate anaemia and iron deficiency. Thus, it is justifiable to question the diagnostic utility of repeated lab tests and to use paediatric tubes and closed flush systems for arterial lines and central venous catheters in such cases [32].

In procedures with a high bleeding risk and in the absence of contraindications, intraoperative blood salvage techniques reduce the need for transfusion in the perioperative phase [33]. The routine use of such techniques helps minimise irreversible blood loss and can improve the surgical outcome [34]. In cancer patients with massive bleeding, cell salvage may be an option, if the technique is available and the recovered blood is leucodepleted and/or irradiated [8].

Depending on the pathology, minimally invasive surgical techniques and meticulous surgical haemostasis are recommended.

Recommendation 7. Implementing a protocol for acute haemorrhage management

- A protocol for ensuring a multidisciplinary approach of acute haemorrhage is recommended to be introduced in every hospital where high bleeding risk surgical procedures are performed.

Implementation Assessment 
- The percentage of surgical patients with a high bleeding risk for whom the acute haemorrhage protocol was used.

The acute haemorrhage protocol must include algorithms for detecting and stopping haemorrhage in various clinical contexts (trauma, surgery, gastroenterology, obstetrics, or transplant). Perioperative algorithms include early surgical haemostasis control, using interventional radiology and endoscopic techniques, packed red blood cells, plasma and platelet concentrates, as well as concentrates of coagulation factors and pharmacological agents in adequate quantities, guided as early as possible by lab or point-of-care haemostasis tests. Perioperative acute haemorrhage protocols must be built upon the European perioperative and traumatic bleeding management guidelines [20,30].

\section{Optimisation of Anaemia Tolerance and Rational Use of Blood Products}

Recommendation 8. Optimising tolerance of anaemia

- Early haemodynamic stabilisation and maintenance of normovolemia are recommended during the entire perioperative period. Advanced haemodynamic monitoring methods may be used to achieve these goals.

- Hyperoxia $\left(\mathrm{PaO}_{2}\right.$ up to $\left.200 \mathrm{mmHg}-26.66 \mathrm{kPa}\right)$ may be used in massively bleeding patients, when blood transfusion is not readily available.

- An adequate anaesthetic technique that reduces intraoperative oxygen consumption (adequate depth and muscle relaxation, tachycardia avoidance) is recommended in all patients. We suggest the routine use of anaesthesia, analgesia and muscle relaxation monitoring methods.

Implementation Assessment

- The percentage of patients for whom advanced haemodynamic monitoring was used, from surgical patients with high bleeding risk.

- The percentage of patients for whom hyperoxia was used, from bleeding surgical patients.

- The percentage of patients for whom the depth of anaesthesia or the neuromuscular block was monitored, from surgical patients with a high bleeding risk.

The patient's tolerance of anaemia can be increased by maintaining normovolemia, haemodynamic optimisation, enhancing oxygen delivery and decreasing oxygen consumption $[35,36]$.

For normovolemia and haemodynamic optimisation, extracellular fluid loss can be replaced with isotonic crystalloid solutions, but their administration must be based on protocols, as for any other drug. Preload and cardiac output optimisation requires advanced haemodynamic monitoring [20].

The fraction of inspired oxygen may be increased to avoid hypoxemia in massively bleeding patients, but extreme hyperoxia $\left(\mathrm{PaO}_{2}>200 \mathrm{mmHg}-26.66 \mathrm{kPa}\right)$ is contraindicated $[20,30]$.

General anaesthesia reduces the patient's need for oxygen, increasing tolerance of anaemia. Moreover, animal research data suggests that effective muscle relaxation improves the tolerance of anaemia [36]. On the other hand, excessive anaesthetic depth is associated with postoperative cognitive dysfunction [37] and should be routinely monitored [38].

Recommendation 9. Restrictive transfusion protocols

- A restrictive transfusion protocol is recommended in the perioperative period. Filling-in a questionnaire on the need of transfusion (APPENDIX 4) for each blood unit order may help rationalising the request for blood products.

Implementation Assessment

- The percentage of patients for whom a restrictive transfusion protocol was used, from the total surgical patients who received transfusion.

- The percentage of patients for whom the questionnaire on transfusion indication (APPENDIX 4) was used, from the total surgical patients who received transfusion.

The rational use of blood products is essential for improving the quality of medical care provided to surgical patients. Besides the previous recommendations, a way to rationalise the use of blood products is to implement a restrictive transfusion protocol [17].

The use of haemoglobin level as an indicator of the need for red cell transfusion is controversial. It has recently been demonstrated that restrictive transfusion protocols can reduce perioperative morbidity (acute neurological events, acute kidney injury, and infection rate) [8], being recommended by both perioperative and traumatic bleeding management guidelines [20,30]. However, for implementing them, it is important to carefully assess each case, as early as the preoperative period, taking into account the haemoglobin level, comorbidities, and tolerance of estimated intraoperative blood loss. A useful tool in assessing the need for transfusion is showed in APPENDIX 4. The questionnaire should accompany each order for blood components.

\section{PBM Programme Monitoring and Evaluation Benchmarks}

Recommendation 10. PBM Programme implementation and monitoring 
- To identify areas of improvement, PBM Programme monitoring benchmarks must be set out in detail for each hospital department.

- For outcome assessment, we recommend that an institutional audit (internal and external) be conducted after benchmarking.

Implementation Assessment

- The percentage of patients with APPENDIX 5 to the clinical observation chart (COC) filled out in full - from the total patients included in the PBM Programme

- The percentage of hospital departments having monitored PBM benchmarks

The impact of the PBM Programme must be assessed after a predefined period, in order to measure and publicise the outputs set out in this document. All participating institutions will conduct a comparative audit for outcome assessment after Programme implementation. The monitoring chart is described in APPENDIX 6.

The key performance indicators to be evaluated are [39]:

- Transfusion rate (percent of transfused patients out of the total elective surgery patients)

- Transfusion index (average number of units of blood products transfused per patient, in patients undergoing elective surgery)

- Haemoglobin values (preoperatively, immediately before surgery, immediately postoperatively and at discharge)

- In-hospital mortality (at 30 days)

- Postoperative infection rate

- Reintervention rate

- Hospital length of stay for patients undergoing elective surgery

- Length of stay in the post-anaesthesia care unit

- Hospital readmission rate

- Cost evolution after implementation of PBM.

The above ten recommendations for implementation of the PBM Programme represent good clinical practice and could be included in the future criteria for accreditation of hospitals in Romania.

\section{Conflict of interest}

Nothing to declare

\section{References}

1. Baron DM, Hochrieser H, Posch M, Metnitz B, Rhodes A, Moreno RP, et al; European Surgical Outcomes Study (EuSOS) group for Trials Groups of European Society of Intensive Care Medicine; European Society of Anaesthesiology. Preoperative anaemia is associated with poor clinical outcome in non-cardiac surgery patients. Br J Anaesth 2014; 113: 416-423. doi: 10.1093/ bja/aeu098
2. Musallam KM, Tamim HM, Richards T, Spahn DR, Rosendaal FR, Habbal A, et al. Preoperative anaemia and postoperative outcomes in non-cardiac surgery: a retrospective cohort study. Lancet 2011; 378: 1396-1407. doi: 10.1016/S0140-6736(11) 61381-0

3. Whitlock EL, Kim H, Auerbach AD. Harms associated with single unit perioperative transfusion: retrospective population based analysis. BMJ 2015; 350: h3037. doi: 10.1136/bmj.h3037

4. World Health Organisation. Global Forum for Blood Safety: Patient Blood Management: Priorities for Action. [cited 2017 May 21]. Available from: http://www.who.int/bloodsafety/ collaboration/who_gfbs_2011_03_priorities_for_action. pdf?ua $=1 \mathrm{~h}$

5. Meybohm P, Richards T, Isbister J, Hofmann A, Shander A, Goodnough LT, et al. Patient Blood Management Bundles to Facilitate Implementation. Transfus Med Rev 2017; 31: 6271. doi: $10.1016 /$ j.tmrv.2016.05.012

6. Vaglio S, Prisco D, Biancofiore G, Rafanelli D, Antonioli P, Lisanti $\mathrm{M}$, et al. Recommendations for the implementation of a Patient Blood Management programme. Application to elective major orthopaedic surgery in adults. Blood Transfus 2016; 14: 23-65. doi: 10.2450/2015.0172-15

7. Gombotz H, Zacharowski K, Spahn DR. Patient Blood Management: Individual Treatment Concept to Reduce and Avoid Anemia. Stuttgart: Georg Thieme Verlag; 2013.

8. Zacharowski K, Spahn DR. Patient blood management equals patient safety. Best Pract Res Clin Anaesthesiol 2016; 30: 159169. doi: 10.1016/j.bpa.2016.04.008

9. Spahn DR, Zacharowski K. Non-treatment of preoperative anaemia is substandard clinical practice. Br J Anaesth 2015; 115: 1-3. doi: 10.1093/bja/aev09

10. Hofmann A, Farmer S, Shander A. Five drivers shifting the paradigm from product-focused transfusion practice to patient blood management. Oncologist 2011; 16(Suppl 3): 3-11. doi: 10.1634/theoncologist.2011-S3-3

11. Liumbruno GM, Vaglio S, Grazzini G, Spahn DR, Biancofiore G. Patient blood management: a fresh look at a fresh approach to blood transfusion. Minerva Anestesiol 2015; 81: 1127-1137

12. Theusinger OM, Kind SL, Seifert B, Borgeat L, Gerber C, Spahn DR. Patient blood management in orthopaedic surgery: a fouryear follow-up of transfusion requirements and blood loss from 2008 to 2011 at the Balgrist University Hospital in Zurich, Switzerland. Blood Transfus 2014; 12: 195-203. doi: 10.2450/ 2014.0306-13

13. Goodnough LT, Shieh L, Hadhazy E, Cheng N, Khari P, Maggio P. Improved blood utilization using real-time clinical decision support. Transfusion 2014; 54: 1358-1365. doi: 10.1111/trf. 12445

14. Gross I, Seifert B, Hofmann A, Spahn DR. Patient blood management in cardiac surgery results in fewer transfusions and better outcome. Transfusion 2015; 55: 1075-1081. doi: 10.1111/ trf. 12946

15. Trentino KM, Farmer SL, Swain SG, Burrows SA, Hofmann A, Ienco $\mathrm{R}$, et al. Increased hospital costs associated with red blood cell transfusion. Transfusion 2015; 55: 1082-1089. doi: 10.1111/ $\operatorname{trf} .12958$

16. Shander A, Isbister J, Gombotz H. Patient blood management: the global view. Transfusion 2016; 56 Suppl 1: S94-102. doi: $10.1111 /$ trf. 13529

17. Meybohm P, Herrmann E, Steinbicker AU, Wittmann M, Gruenewald M, Fischer D, et al. Patient Blood Management is associated with a substantial reduction of red blood cell utilization and safe for patient's outcome: A prospective, multicenter cohort 
study with a noninferiority design. Ann Surg 2016; 264: 203 211. doi: $10.1097 /$ SLA.0000000000001747

18. Union Européenne des Médecins Spécialistes / European Union of Medical Specialists. Training Requirements for the Specialty of Anaesthesiology, Pain and Intensive Care Medicine: European Standards of Postgraduate Medical Specialist Training [Internet]. Brussels: UEMS; s.a. [cited 2017 May 21]. Available from: http:/ /www.eba-uems.eu/resources/PDFS/Training/AnaesthesiologyTraining-Requirements-March-2013.pdf

19. Muńoz M, Acheson AG, Auerbach M, Besser M, Habler O, Kehlet $\mathrm{H}$, et al. International consensus statement on the peri-operative management of anaemia and iron deficiency. Anaesthesia 2017; 72: 233-247. doi:10.1111/anae.13773

20. Kozek-Langenecker SA, Ahmed AB, Afshari A, Albaladejo P, Aldecoa C, Barauskas G, et al. Management of severe perioperative bleeding: guidelines from the European Society of Anaesthesiology: First update 2016. Eur J Anaesthesiol 2017; 34: 332-395. doi: 10.1097/EJA.0000000000000630

21. World Health Organization. Haemoglobin concentrations for the diagnosis of anaemia and assessment of severity: Vitamin and Mineral Nutrition Information System. (WHO/NMH/NHD/ MNM/11.1) [Internet]. Geneva: World Health Organization; 2011 [cited 2016 April 28]. Available from: http://www.who.int/ vmnis/indicators/ haemoglobin.pdf

22. Muńoz M, Gómez-Ramírez S, Martín-Montańez E, Pavía J, Cuenca J, García-Erce JA. Perioperative intravenous iron: an upfront therapy for treating anaemia and reducing transfusion requirements. Nutr Hosp 2012; 27: 1817-1836. doi: 10.3305/ nh.2012.27.6.6087

23. Ganzoni AM. Eisen-Dextran intravenös: therapeutische und experimentelle Möglichkeiten. Schweiz Med Wochenscr 1970; 100: 301-303

24. Maynor L, Brophy DF. Risk of infection with intravenous iron therapy. Ann Pharmacother 2007; 41: 1476-1480. doi: 10.1345/ aph. $1 \mathrm{~K} 187$

25. Siriam S, Xenocostas A, Lazo-Langner A. Erythropoietin in anaemia of unknown etiology: A systematic review and metaanalysis. Hematology 2016; 21: 234-240. doi: 10.1080/ 10245332.2015.1101972

26. Haas T, Fries D, Tanaka KA, Asmis L, Curry NS, Schöchl H. Usefulness of standard plasma coagulation tests in the management of perioperative coagulopathic bleeding: is there any evidence? Br J Anaesth 2015; 114: 217-224. doi: 10.1093/bja/ aeu303

27. Koscielny J, Ziemer S, Radtke H, Schmutzler M, Pruss A, Sinha $\mathrm{P}$, et al. A practical concept for preoperative identification of patients with impaired primary haemostasis. Clin Appl Thromb Hemost 2004; 10: 195-204. doi: 10.1177/ 107602960401000301

28. De Hert S, Imberger G, Carlisle J, Diemunsch P, Fritsch G, Moppett I, et al; Task Force on Preoperative Evaluation of the Adult Noncardiac Surgery Patient of the European Society of Anaesthesiology. Preoperative evaluation of the adult patient undergoing non-cardiac surgery: guidelines from the European Society of Anaesthesiology. Eur J Anaesthesiol 2011; 28: 684722. doi: 10.1097/EJA.0b013e3283499e3b
29. Weber CF, Görlinger K, Meininger D, Herrmann E, Bingold T, Moritz A, et al. Point-of-care testing: a prospective, randomized clinical trial of efficacy in coagulopathic cardiac surgery patients. Anesthesiology 2012; 117: 531-547. doi: 10.1097/ALN. 0b013e318264c644

30. Rossaint R, Bouillon B, Cerny V, Coats TJ, Duranteau J, Fernández-Mondéjar E, et al. The European guideline on management of major bleeding and coagulopathy following trauma: fourth edition. Crit Care 2016; 20: 100. doi: 10.1186/ s13054-016-1265-x

31. Koch CG, Reineks EZ, Tang AS, Hixson ED, Phillips S, Sabik JF $3^{\text {rd }}$, et al. Contemporary bloodletting in cardiac surgical care. Ann Thorac Surg 2015; 99: 779-784. doi: 10.1016/ j.athoracsur.2014.09.062

32. Fischer DP, Zacharowski KD, Meybohm P. Savoring every drop - vampire or mosquito? Crit Care 2014; 18: 306. doi: 10.1186/ cc13884

33. Wang G, Bainbridge D, Martin J, Cheng D. The efficacy of an intraoperative cell saver during cardiac surgery: a meta-analysis of randomized trials. Anesth Analg 2009; 109: 320-330. doi: 10.1213/ane.0b013e3181aa084c

34. Meybohm P, Choorapoikayil S, Wessels A, Herrmann E, Zacharowski K, Spahn DR. Washed cell salvage in surgical patients: A review and meta-analysis of prospective randomized trials under PRIMSA. Medicine (Baltimore) 2016; 95: e4490. doi: 10.1097/MD.0000000000004490

35. Habler O, Meier J, Pape A, Kertscho H, Zwissler B. Tolerance to perioperative anemia. Mechanisms, influencing factors and limits. Anaesthesist 2006; 55: 1142-1156. doi: 10.1007/s00101006-1055-y

36. Meier J, Gombotz H. Pillar III - Optimisation of anaemia tolerance. Best Pract Res Clin Anaesthesiol 2013; 27: 111-119. doi: 10.1016/j.bpa.2013.02.005

37. Soehle M, Dittmann A, Ellerkmann RK, Baumgarten G, Putensen $\mathrm{C}$, Guenther U. Intraoperative burst suppression is associated with postoperative delirium following cardiac surgery: a prospective, observational study. BMC Anesthesiol 2015; 15: 61. doi: 10.1186/s12871-015-0051-7

38. Aldecoa C, Bettelli G, Bilotta F, Sanders RD, Audisio R, Borozdina A, et al. European Society of Anaesthesiology evidence-based and consensus-based guideline on postoperative delirium. Eur $\mathrm{J}$ Anaesthesiol 2017; 34: 192-214. doi: 10.1097/EJA. 0000000000000594

39. Gombotz H, Hofmann A, Nørgaard A, Kastner P; European Comission (Directorate-General for Health and Food Safety). Supporting Patient Blood Management (PBM) in the EU - A practical implementation guide for hospitals : Technical Report, March 2017. Luxembourg: Publications Office of the European Union; 2017. doi: $10.2818 / 533179$

40. Jammer I, Wickboldt N, Sander M, Smith A, Schultz M, Pelosi P, et al. Standards for definitions and use of outcome measures for clinical effectiveness research in perioperative medicine: European Perioperative Clinical Outcome (EPCO) definitions : A statement from the ESA-ESICM joint taskforce on perioperative outcome measures. Eur J Anaesthesiol 2014; 32: 88-105. doi: 10.1097/EJA.0000000000000118 


\section{APPENDICES}

\section{Appendix 1}

\section{High Bleeding Risk Surgery}

\section{General Surgery}

- Hemihepatectomy

- Adrenalectomy

- Oesophagectomy

- Duodenopancreatectomy

- Gastrectomy

- Hemicolectomy

- Splenectomy

- Other major abdominal, pelvic or thoracic surgery

\section{Vascular Surgery}

- Large vessels surgery

- Lower limb amputation

\section{Neurosurgery}

- Osteosynthesis, open repositioning and spinal osteoplasty

- Craniotomy, incision of brain and/or cerebral meninges

\section{Orthopaedics-Traumatology}

- Pelvic and hip joint surgery

- Spinal surgery

- Flap surgery

- Lower limb surgery (osteosynthesis, osteotomy)

- Polytrauma patients

- Upper limb surgery (endoprosthesis, osteosynthesis, osteotomy)

\section{Urology}

- Cystectomy

- Nephrectomy (including partial)

- Open prostatectomy

\section{Cardiac Surgery}

- Procedures requiring extracorporeal circulation

- Pericardectomies 


\section{Appendix 2}

\section{Indications for Iron Administration}

Iron dosing is based on the haemoglobin level and body weight. Depending on the amount of blood loss, one of the following methods is used:

a) The Ganzoni formula (of historical interest) [23]

Iron deficit $(\mathrm{mg})=$ Body weight $(\mathrm{kg}) \times($ Target $\mathrm{Hb}-$ Actual $\mathrm{Hb}) \times 2.4+$ Iron stores

- Body weight: in case of extreme obesity/oedema, the ideal body weight must be used, which is worked out based on a body mass index of 25 . The ideal body weight $(\mathrm{kg})=[\text { Height }(\mathrm{m})]^{2} \times 25$

- The 2.4 quotient results from the following formula: haemoglobin-contained iron $(0.34 \%) \times$ blood volume $(7 \%$ of body weight $) \times$ haemoglobin conversion factor into $\mathrm{mg} / \mathrm{L}(10,000)=0.0034 \times 0.07 \times$ 10,000 .

- Minimum iron stores: $500 \mathrm{mg}$ (or $10-15 \mathrm{mg} / \mathrm{kg}$ ).

b) The simplified formula (currently recommended)

\begin{tabular}{lllll}
\hline \multicolumn{2}{c}{ Haemoglobin } & \multicolumn{3}{c}{ Patient's body weight } \\
$\mathrm{g} / \mathrm{dL}$ & $\mathrm{mmol} / \mathrm{L}$ & $<35 \mathrm{~kg}$ & $35-70 \mathrm{~kg}$ & $>70 \mathrm{~kg}$ \\
\hline$<10$ & $<6.2$ & $500 \mathrm{mg}$ & $1500 \mathrm{mg}$ & $2000 \mathrm{mg}$ \\
$10-14$ & $6.2-8.7$ & $500 \mathrm{mg}$ & $1000 \mathrm{mg}$ & $1500 \mathrm{mg}$ \\
$>14$ & $>8.7$ & $500 \mathrm{mg}$ & $500 \mathrm{mg}$ & $500 \mathrm{mg}$ \\
\hline
\end{tabular}

c) When blood loss can be estimated: $200 \mathrm{mg}$ of iron for each blood loss unit $(450 \mathrm{~mL}$ )

d) Using the post-bleed haemoglobin decrease: the Ganzoni equation without the iron needed for storage: Iron dose $(\mathrm{mg})=$ Body weight $(\mathrm{kg}) \times($ Target $\mathrm{Hb}-$ Actual $\mathrm{Hb}) \times 2.4$.

\section{Route and rhythm of administration}

- The maximum dose is $20 \mathrm{mg} / \mathrm{kg}$ but no more than $1000 \mathrm{mg}$ at once.

- The minimum time gap between two doses is 7 days.

For i.v. administration the iron product should be diluted in $0.9 \% \mathrm{NaCl}$ solution exclusively. Mixing it with other drugs is prohibited. The solution must not be excessively diluted because the preparation is unstable in dilutions lower than $2 \mathrm{mg} / \mathrm{mL}$.

- i.v. bolus/infusion must be slow (15-30 min depending on quantity and dilution). Please follow the instructions provided by producers of the iron i.v. preparations. 


\section{Appendix 3}

\section{Haemostasis Disorder Screening Questionnaire (amended based on reference 6)}

Will be filled by the patient and validated by the physician

\begin{tabular}{|c|c|c|c|c|}
\hline \multicolumn{2}{|r|}{ Please tick or fill in the answer (Yes or No): } & No & Yes & Validation \\
\hline \multicolumn{5}{|c|}{ Have you ever been diagnosed with a kidney, liver or bone marrow disease? } \\
\hline \multicolumn{2}{|r|}{ Have you ever noticed any of the following types of bleeds, sometimes without an apparent cause? } & & & \\
\hline a) & Nosebleeds (without other causes like prolonged sneezing, dry air, blowing your nose too hard, etc.) & & & \\
\hline b) & $\begin{array}{l}\text { Large bruises or bleeding spots (also on the trunk, occurring even in the absence of trauma/hits); } \\
\text { provide diameter. }\end{array}$ & & & \\
\hline c) & Bleeding joints, soft tissue or muscles & & & \\
\hline \multicolumn{5}{|c|}{ Have you ever had prolonged bleeding in case of cuts or scratches? } \\
\hline \multicolumn{5}{|c|}{ Do you remember having severe or prolonged bleeding after tooth extractions? } \\
\hline \multicolumn{5}{|c|}{ Do you remember having prolonged bleeding during or after surgery? } \\
\hline \multicolumn{5}{|c|}{ Do your wounds take a lot of time to heal? } \\
\hline \multicolumn{5}{|c|}{ Have you ever had cases of haemophilia or bleeding disorders in your family? } \\
\hline \multicolumn{5}{|c|}{$\begin{array}{l}\text { Do you take or have you taken blood thinners lately? (e.g. Sintrom, Trombostop, Pradaxa, Eliquis, } \\
\text { Xarelto, Aspenter, Plavix, Brilique, Efient, etc.) }\end{array}$} \\
\hline \multicolumn{5}{|c|}{$\begin{array}{l}\text { Are you currently on pain killers or anti-rheumatics, even over-the-counter ones? (e.g. Aspirin, Voltaren, } \\
\text { Ibuprofen, etc.) }\end{array}$} \\
\hline \multicolumn{2}{|r|}{ Have you ever had an abnormal coagulation test or anaemia? } & & & \\
\hline \multicolumn{5}{|c|}{ Additional questions for women: } \\
\hline \multicolumn{2}{|r|}{ Do you have a long ( $>7$ days) and/or heavier (frequent tampon/pad change) period? } & & & \\
\hline \multicolumn{2}{|r|}{ Have you had massive bleeding after childbirth or during your period? } & & & \\
\hline
\end{tabular}

[A positive answer to any of these questions requires a thorough haemostatic investigation and specialist consultations (cardiology, haemostasis)]

Signature of physician validating questionnaire

\section{Appendix 4}

\section{Blood Transfusion Need Assessment Questionnaire (Frankfurt University Hospital SOP, with approval)}

1. $\mathrm{Hb}<6 \mathrm{~g} / \mathrm{dL}$

1.a. $\square$ Irrespective of compensatory capacity

2. Hb 6-8 g/dL

2.a. $\square$ Indicators of anaemic hypoxia (tachycardia, hypotension, ischaemia on ECG, lactic acidosis)

2.b. $\square$ Reduced compensatory capacity, presence of risk factors (coronary disease, heart failure, cerebrovascular diseases)

2.c. $\square$ Other indications

3. $\mathrm{Hb} \mathrm{8-10} \mathrm{g} / \mathrm{dL}$

3.a. $\square$ Indicators of anaemic hypoxia (tachycardia, hypotension, ECG ischemia, lactic acidosis)

3.b. $\square$ Other indications

4. $\mathrm{Hb}>10 \mathrm{~g} / \mathrm{dL}$

4.a. $\square$ Other indications 


\section{Appendix 5}

Patient Blood Management Programme Monitoring and Evaluation Benchmarks

\begin{tabular}{|l|l|l|l|l|l|l|}
\hline $\mathrm{COC}^{*}$ & & Date of admission & & Age & & Gender (M/F) \\
\hline
\end{tabular}

* COC - clinical observation chart

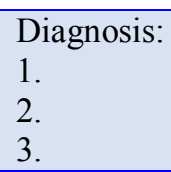

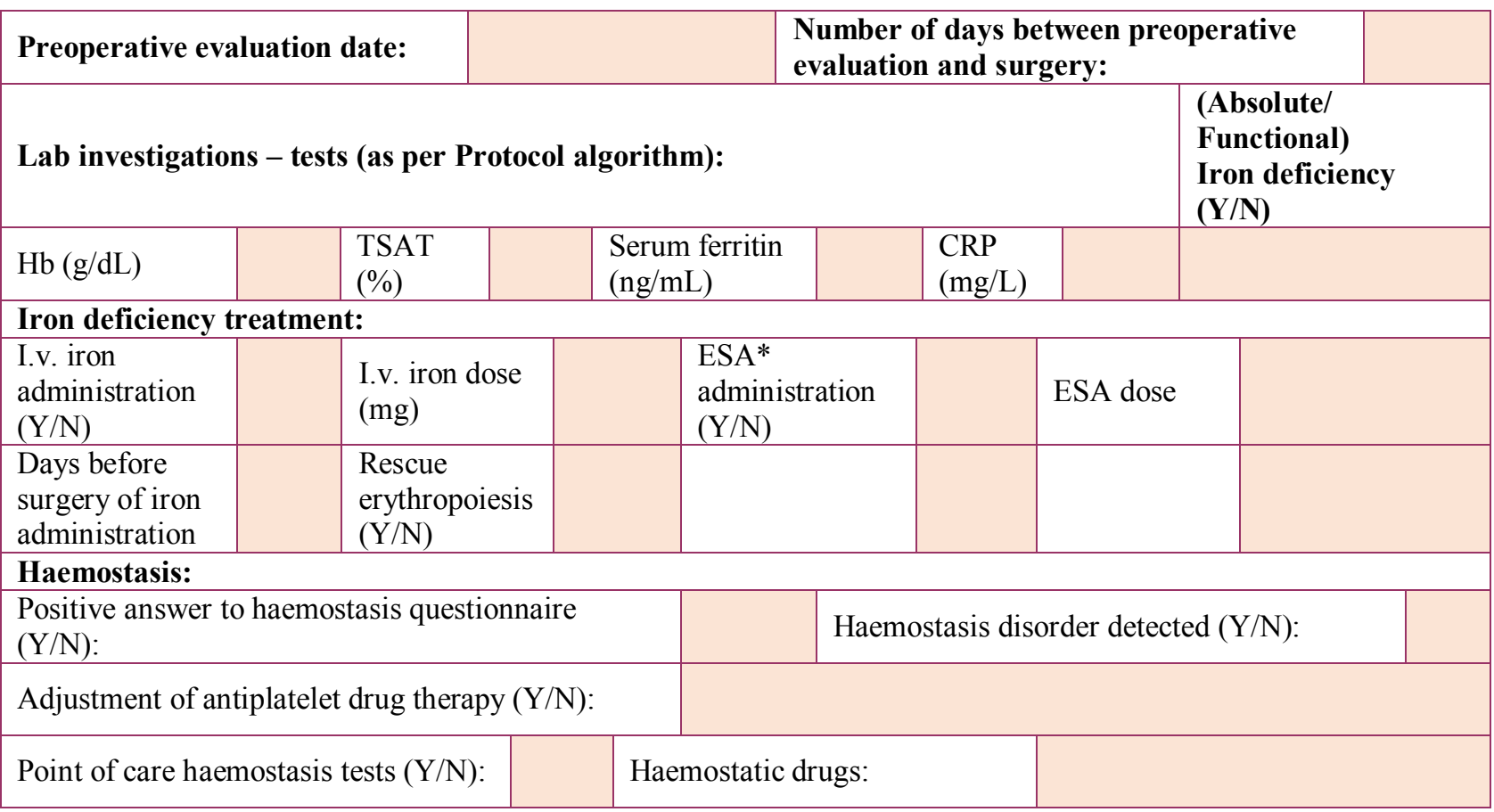

* ESA - Erythropoiesis stimulating agents

\begin{tabular}{|l|l|l|}
\hline \multicolumn{2}{|l|}{ Surgery: } \\
\hline Type of surgery & \multicolumn{2}{|l|}{} \\
\hline Date of surgery & & \\
\hline
\end{tabular}

\begin{tabular}{|l|l|l|l|l|l|}
\hline Length of stay in hospital (days): & & Length of stay in ICU (days): & & In-hospital death (Y/N) \\
\hline
\end{tabular}

\begin{tabular}{|l|l|l|l|l|l|}
\hline \multicolumn{4}{|l|}{ Haemoglobin $(\mathrm{Hb}, \mathrm{g} / \mathrm{dL})$ : } \\
\hline At first preoperative evaluation: & & Preoperative: & & Postoperative: \\
\hline At discharge & & & \\
\hline
\end{tabular}


Blood loss minimisation:

\begin{tabular}{|l|l|l}
\hline Use of micro tubes for & & Use of closed flush systems
\end{tabular} specimen collection $(\mathrm{Y} / \mathrm{N})$ : for arterial lines and central venous catheters $(\mathrm{Y} / \mathrm{N})$ :

Use of blood salvage systems $(\mathrm{Y} / \mathrm{N})$ :

Acute haemorrhage $(\mathrm{Y} / \mathrm{N})$ :

Use of the multidisciplinary protocol for acute haemorrhage $(\mathrm{Y} / \mathrm{N})$ :

Optimisation of the patient's tolerance of anaemia:

Use of advanced haemodynamic monitoring $(\mathrm{Y} / \mathrm{N})$ :

Use of hyperoxia $(\mathrm{Y} / \mathrm{N})$ :

Monitoring of anaesthetic depth or neuromuscular block $(\mathrm{Y} / \mathrm{N})$ :

\begin{tabular}{|l|l|l|l|l|l|l|}
\hline Transfusions (number of units for each component): & Preoperative: & & Intraoperative: & & Postoperative: & \\
\hline Packed red blood cells & Preoperative: & & Intraoperative: & & Postoperative: & \\
\hline Platelet concentrate & Preoperative: & & Intraoperative: & & Postoperative: & \\
\hline Fresh frozen plasma & Preoperative: & & Intraoperative: & & Postoperative: & \\
\hline Other blood component: & & & & & & \\
\hline
\end{tabular}

\begin{tabular}{|c|c|c|c|}
\hline Postoperative complications & YES & NO & \\
\hline \multicolumn{3}{|c|}{ General postoperative complications - during hospital stay (Y/N), see Glossary: } & Comments \\
\hline Acute kidney injury & & & Stage: \\
\hline \multicolumn{4}{|l|}{ Major adverse cardiac events (MACE) } \\
\hline \multicolumn{4}{|l|}{ Myocardial infarction } \\
\hline \multicolumn{4}{|l|}{ Pulmonary embolism } \\
\hline \multicolumn{4}{|l|}{ Pneumonia } \\
\hline \multicolumn{4}{|l|}{ Postoperative haemorrhage } \\
\hline \multicolumn{4}{|l|}{ Stroke } \\
\hline \multicolumn{4}{|l|}{ Surgical site infection (deep) } \\
\hline \multicolumn{4}{|l|}{ Surgical site infection (superficial) } \\
\hline Other complications (based on the type of surgery): & YES & NO & Comments \\
\hline \multicolumn{4}{|l|}{$\begin{array}{l}- \\
-\end{array}$} \\
\hline Transfusion-related complications & & & \\
\hline
\end{tabular}

Note: Complications reported in Appendix 5 are defined according to reference [40] 


\section{Appendix 6}

\section{Patient Blood Management Protocol Implementation Monitoring Chart}

HOSPITAL:

PBM Implementation Group Coordinator:

Monitoring Period:

Recommendation 1. Setting up a PBM Implementation Group (IG)

\begin{tabular}{|l|l|}
\hline Number of PBM medical education events held & \\
\hline Number of reports from the hospital PBM IG & \\
\hline
\end{tabular}

Recommendation 2. Planning of the surgical intervention

Total number of patients with high bleeding risk elective procedures

The percentage of patients examined by anaesthesiologists more than a week before surgery, from the total number of patients who underwent surgical procedures with a high bleeding risk.

Recommendation 3. Assessing anaemia and iron deficiency

The percentage of patients with anaemia and iron deficiency (absolute or functional) identified at least one week before surgery from those who underwent surgical procedures with a high bleeding risk.

Recommendation 4. Optimising patient red cell mass

The percentage of patients with iron deficiency (absolute and functional) in whom iron was administered before surgery from the total number of iron deficient patients undergoing with high bleeding risk.

The percentage of patients in whom rescue erythropoiesis was undertaken from the total number of iron deficient patients undergoing surgery with a high bleeding risk.

Recommendation 5. Assessing haemostatic abnormalities

The percentage of patients with a haemostasis abnormality risk assessment questionnaire filled in, from the total number of patients with high bleeding risk surgery.

The percentage of patients with positive answers to the questionnaire, in whom haemostatic abnormalities were detected, from the total number of patients with a positive answer.

The percentage of patients with anticoagulant and/or antiplatelet agents adjusted perioperatively, from the total number of patients treated with anticoagulants and/or antiplatelet drugs who underwent procedures with a high bleeding risk.

The percentage of patients in whom point-of-care tests were used to detect perioperative haemostasis abnormalities, from the total number of patients who underwent procedures with a high bleeding risk.

The percentage of patients who received tranexamic acid prophylactically or as a treatment, from the total number of patients who underwent procedures with a high bleeding risk. 
Recommendation 6. Minimising iatrogenic blood loss

The percentage of patients for whom micro tubes were used, from all investigated patients who underwent surgical procedures with a high bleeding risk.

The percentage of patients for whom closed flush systems were used for arterial lines and central venous catheters, from all patients with arterial lines or central venous catheters who underwent surgical procedures with a high bleeding risk.

The percentage of patients for whom blood salvage (cell-saver) systems were used from patients who underwent surgical procedures with a high bleeding risk.

Recommendation 7. Implementing an acute haemorrhage management protocol

The percentage of surgical patients with high bleeding risk for whom the acute haemorrhage protocol was used.

Recommendation 8. Optimising tolerance of anaemia

The percentage of patients for whom advanced haemodynamic monitoring was used, from surgical patients with high bleeding risk.

The percentage of patients for whom hyperoxia (up to $200 \mathrm{mmHg}-26.66 \mathrm{kPa}$ ) was used, from bleeding surgical patients.

The percentage of patients for whom depth of anaesthesia or neuromuscular blockade was monitored, from surgical patients with high bleeding risk.

Recommendation 9. Restrictive transfusion protocols

The percentage of patients for whom a restrictive transfusion protocol was used, from the total surgical patients who received transfusion.

The percentage of patients for whom the questionnaire on transfusion indication (APPENDIX 4) was used, from the total surgical patients who received transfusion.

Recommendation 10. PBM programme implementation and monitoring

The percentage of patients with APPENDIX 5 to the clinical observation chart (COC) filled out in full from the total patients included in the PBM Programme

The Percentage of hospital departments having monitored PBM benchmarks

A comparative audit was conducted for outcome assessment after programme implementation $(\mathrm{Y} / \mathrm{N})$ 


\section{Glossary}

Blood components - human blood constituents, separated from the whole blood (packed red blood cells, plasma, platelet concentrate, cryoprecipitate), plasma or platelets collected through apheresis, cryoprecipitate-used for therapeutic purposes, defined under the Order of the Minister of Public Health No 1237 of 10 July 2007 approving the National Classification of Human Blood Components for Therapeutic Use and in the National Guidelines for the Therapeutic and Reasonable Use of Blood and Human Blood Components.

Absolute iron deficiency - reduced iron stores (low serum ferritin) and decreased iron available for erythropoiesis (low transferrin saturation). Iron deficiency may occur in the absence of anaemia.

Functional iron deficiency - decreased iron available for erythropoiesis (low transferrin saturation), but iron stores are present (normal or high serum ferritin). It may be caused by:

- Rapid increase in iron consumption for erythropoiesis, for example during treatment with erythropoiesis-stimulating agents;

- Overly slow mobilisation of stored iron, due to inflammation.

Serum ferritin - indicator of iron stores (the serum level is directly proportional to ferritin containing iron from intracellular stores). Since serum ferritin is a positive acute phase reactant, levels over $100 \mathrm{ng} / \mathrm{mL}$ may indicate either adequate iron stores, or inflammation.

Transferrin - an iron-carrying protein. It provides the only means for iron to get from stores to erythrocyte-producing bone marrow cells. Hence, the amount of carried transferrin-bound iron - expressed as transferrin saturation - TSAT - indicates how much iron is available for erythropoiesis.

eGFR - estimated glomerular filtration rate, worked out based on the MDRD or CKD-EPI formulae.

Acute kidney injury - Kidney Disease Improving Global Outcomes (KDIGO) guidelines.

Stage 1 - serum creatinine 1.5-1.9 times baseline values within 7 days, or $0.3 \mathrm{mg} / \mathrm{dl}$ increase within $48 \mathrm{~h}$, or urine output $<0,5 \mathrm{ml} / \mathrm{kg} / \mathrm{min}$ for $6-12 \mathrm{~h}$.

Stage 2-2.0-2.9 times baseline value within 7 days, or $<0.5 \mathrm{ml} / \mathrm{kg} / \mathrm{min}$ for $12 \mathrm{~h}$

Stage 3 - 3.0 times baseline value within 7 days, or increase in serum creatinine to $>4 \mathrm{mg} / \mathrm{dl}$ with an acute rise of more than $0.5 \mathrm{mg} / \mathrm{dl}$, or initiation of renal replacement therapy, or anuria for $12 \mathrm{~h}$.

Major adverse cardiac events (MACE) - defined as one of the following:

- Non-fatal cardiac arrest. An absence of cardiac rhythm or presence of chaotic rhythm requiring any component of basic or advanced life support.

- Acute myocardial infarction (see below).

- Congestive heart failure. New in-hospital signs or symptoms of dyspnoea or fatigue, orthopnoea, paroxysmal nocturnal dyspnoea, increased jugular venous pressure, pulmonary rales on physical examination, cardiomegaly or pulmonary vascular engorgement.

- New cardiac arrhythmia. ECG evidence of atrial flutter, atrial fibrillation, or second- or third-degree atrioventricular conduction block.

- Angina. Dull diffuse substernal chest discomfort precipitated by exertion or emotion and relieved by rest or glyceryl trinitrate.

Myocardial infarction - increase in serum cardiac biomarker values (preferably cardiac troponin) with at least one value above the $99^{\text {th }}$ percentile upper reference limit and at least one of the following criteria: symptoms of ischaemia, new or presumed new significant ST segment or T wave ECG changes or new left bundle branch block; development of pathological Q waves on ECG; radiological or echocardiographic evidence of new loss of viable myocardium or new regional wall motion abnormality; identification of an intracoronary thrombus at angiography or autopsy.

Pneumonia - two or more serial chest radiographs with at least one of the following:

- New or progressive and persistent infiltrates

- Consolidation

- Cavitation 
And at least one of the following:

- Fever (>38 Celsius) with no other recognised cause

- Leucopaenia (white cell count $<4 \times 10^{9} \mathrm{l}^{-1}$ ) or leucocytosis (white cell count $>12 \times 10^{9} \mathrm{l}^{-1}$ )

- For adults $>70$ years old, altered mental status with no other recognised cause

And at least two of the following:

- New onset of purulent sputum or change in character of sputum, or increased respiratory secretions, or increased suctioning requirements.

- New onset of worsening cough, or dyspnoea, or tachypnoea.

- Rales or bronchial breath sounds.

- Worsening gas exchange (hypoxaemia, increased oxygen requirement, increased ventilator demand).

Postoperative haemorrhage - blood loss within $72 \mathrm{~h}$ after the start of surgery which would normally result in transfusion of blood.

Pulmonary embolism - a new blood clot or thrombus within the pulmonary arterial system.

Stroke - embolic, thrombotic or haemorrhagic cerebral event with persistent residual motor, sensory or cognitive dysfunction.

Surgical site infection (superficial) - an infection which meets the following criteria:

- Infection occurs within 30 days after surgery and

- Involves only skin and subcutaneous tissue of the incision and

- The patient has at least one of the following:

- Purulent drainage from the superficial incision;

- Organisms isolated from an aseptically obtained culture or fluid;

- At least one of the following symptoms or signs of infection: pain or tenderness, localised swelling, redness or heat, and superficial incision is deliberately opened by surgeon and is culture-positive or not cultured;

- Diagnosis of an incisional surgical site infection by a surgeon or attending physician.

Surgical site infection (deep) - an infection that meets the following criteria:

- Infection occurs within 30 days after surgery if no implant is left in place or 1 year if implant is left in place.

- Involves deep soft tissues of the incision.

- The patient has at least one of the following:

- Purulent drainage from the deep incision but not from the organ/space component of the surgical site

- A deep incision spontaneously dehisces or is deliberately opened by a surgeon and is culture-positive or not cultured when the patient has at least one of the following symptoms or signs: fever $\left(>38^{\circ} \mathrm{C}\right)$, or localised pain or tenderness;

- An abscess or other evidence of infection involving the deep incision is found on direct examination, during surgery, or by histopathological or radiological examination;

- Diagnosis of an incisional surgical site infection by a surgeon or attending physician. 